\title{
Annexin 5 decreases the diffusion of lipid and channel molecules in an artificial lipid bilayer*
}

\author{
Takehiko Ichikawa \\ Department of Biophysical Engineering, Graduate School of Engineering Science, \\ Osaka University, 1-3 Yamadaoka, Suita, Osaka 565-0871, Japan \\ Yuko Takeuchi, Takaaki Aoki, and Toru Ide $^{\dagger}$ \\ Creation of Innovative Technology by Integration of Nanotechnology \\ with Information, Biological and Environmental Technologies, JST \\ c/o Laboratory for Nanobiology, Graduate School of Frontier Bioscience, \\ Osaka University, 1-3 Yamada-oka, Suita, Osaka 565-0871, Japan \\ (Received 15 May 2005; Accepted 4 June 2005; Published 18 June 2005)
}

\begin{abstract}
The effects of annexin 5 on the lateral diffusion of single molecule lipids and single molecule proteins were studied in a lipid bilayer membrane. Single $\beta$-BODIPY HPC and ryanodine receptor type 2 (RyR2) labeled with Cy5 molecules were monitored by fluorescence microscopy. The diffusion coefficient was calculated in the presence and absence of annexin 5. The diffusion coefficients of $\beta$-BODIPY HPC and RyR2 were $11 \times 10^{-8} \mathrm{~cm}^{2} / \mathrm{s}$ and $2.7 \times 10^{-8} \mathrm{~cm}^{2} / \mathrm{s}$ in the absence of annexin 5 , respectively. The diffusion coefficients of $\beta$-BODIPY HPC and RyR2 in the presence of $1 \mu \mathrm{M}$ annexin 5 were $2.4 \times 10^{-10} \mathrm{~cm}^{2} / \mathrm{s}$ and $2.6 \times 10^{-10} \mathrm{~cm}^{2} / \mathrm{s}$, respectively. Overall, $1 \mu \mathrm{M}$ annexin 5 decreases the lateral diffusion coefficient 100-500 fold. Regarding RyR2, annexin 5 has little effect on function and can be used to immobilize RyR2 in a lipid bilayer system. [DOI: 10.1380/ejssnt.2005.213]
\end{abstract}

Keywords: Nano-scale imaging, measurement, and manipulation technology; Biological molecules - proteins; Biophysics, medical physics, biomedical engineering; Ion channel

\section{INTRODUCTION}

Recent developments in optical microscopy have enabled us to directly observe single fluorescent molecules. In particular, the single-molecule imaging technique has been used to study a variety of biological molecules as reviewed by Weiss [1]. These techniques also apply to ion channel proteins. Single ion channel current recordings have been performed using the patch-clamp technique or lipid bilayer system. However, imaging of single channel proteins in lipid bilayers is far less established than the technique for single-channel current recordings. Studying single channel proteins, our group has simultaneously observed ions passing trough channels using a horizontal bilayer membrane formed on a thin agarose layer $[2,3]$ and simultaneously recorded the single channel's current. Others have also observed ion movement and single channel current recordings $[4,5]$. However no group has yet reported the structural properties of ion channels by correlating the electrical current and the intermolecular signal. The major obstacle to achieving these observations has been the inability to immobilize the channel proteins. In artificial lipid bilayers, channel proteins can move freely. Because the microscopic field is smaller than the lipid bilayer, channel proteins sometimes move out of the microscopic field. Although the size of lipid bilayer can be shrunk by reducing the hole size at the bottom chamber, the fluorescence of a single molecule disappears due to the florescence generated from the edge of the hole. For this reason the size of lipid bilayer has to be bigger than the

*This paper was presented at International Symposium on Molecule-Based Information Transmission and Reception - Application of Membrane Protein Biofuction- (MB-ITR2005), Okazaki, Japan, 3-7 March, 2005.

$\dagger$ Corresponding author: ide@phys1.med.osaka-u.ac.jp size of the microscopic field, making it difficult to perform long term observations until now.

In 2004, Peng et al. reported that annexin 12 decreases the diffusion of single cardiac ryanodine receptors [6]. By observing the center of the calcium flux of RyR2 at various annexin 12 concentrations, they found that $2 \mu \mathrm{M}$ annexin 12 decreases the diffusion of the lipid bilayer 700 fold compared to that in the absence of annexin 12. We took annexin 5, which is commercially available, and examined whether annexin 5 changes the diffusion coefficient of the lipid bilayer by observing single molecules directly.

Annexins constitute a large family of structurally related proteins, which have the common characteristic of strongly binding to anionic phospholipids in a calcium-

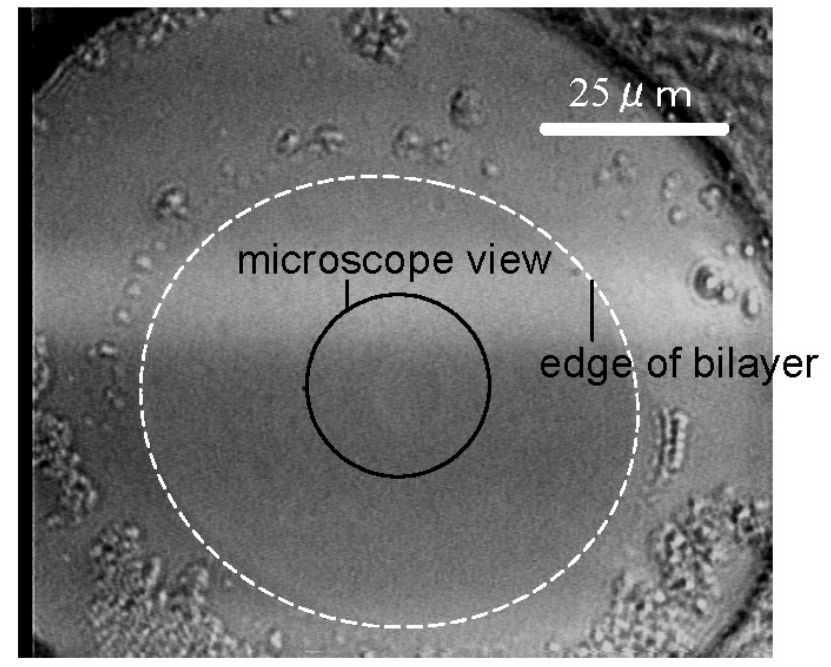

FIG. 1: A top view of the membrane at $0 \mathrm{mV}$. Outer dotted circle is the edge of the bilayer. Inner circle is the microscopic field with $\times 100$ objective lens. 
dependent manner [7-9]. The crystal structure of annexin 5 was solved and refined to $2.0 \AA[10]$. However, there is to date no study that observes single lipid molecules in a lipid bilayer directly under the influence of annexin 5 . In this paper, we report the direct observation of single lipids and channel molecules by annexin 5 in a modified lipid bilayer.

\section{MATERIALS AND METHODS}

\section{A. Reagents}

Phosphatidylethanolamine (PE), Phosphatidylserine (PS) and Phosphatidylcholine (PC) were purchased from Sigma-Aldrich (USA), $\beta$-BODIPY 530/550 HPC from Molecular Probes (USA), n-decane from Wako (Japan), monoclonal anti-ryanodine receptor antibody (antiRyR2) raised in a mouse against a canine cardiac ryanodine receptor (RyR2) from Affinity Bioreagents (USA), and Cy5monofunctional reactive dye from Amersham Pharmacia Biotech (Sweden). All of the other chemicals were commercial products of an analytical grade.

\section{B. Purification of cardiac ryanodine receptor} (RyR2)

The purified canine cardiac RyR2 was prepared according to the method of Anderson et al. [11], and the reconstitution was performed according to the method of Lee et al. [12].

\section{Fluorescence labeling with the Cy5-conjugated antibody}

RyR2 antibody was conjugated to Cy5-monofunctional reactive dye following the appended instructions. Free Cy5 was removed using a NAP-10 gel-filtration column (Amersham Pharmacia Biotech, Sweden). After conjugation with $\mathrm{Cy} 5$, antiRyR2 was reacted with purified RyR2 for 2 hours at $4^{\circ} \mathrm{C}$. In order to remove unreacted antibodies, the labeled sample was washed twice with 5 volumes of the washing buffer following centrifugation in a HITACHI RP65 rotor at 55,000 rpm for 1 hour. After centrifugation, the sample was quickly frozen and stored at $-80^{\circ} \mathrm{C}$.

\section{Microscopy}

The optical system was been previously described in detail $[2,3]$. Figure 2 shows a schematic overview of the lipid bilayer formation apparatus. This apparatus consists of two chambers. Lipid bilayers were formed in a small hole (about $100 \mu \mathrm{m}$ diameter) at the bottom of the upper chamber. The surface of the bilayers touched the agarosecoated cover glass at the bottom of the lower chamber. An oil immersion objective lens (PlanApo, 100×, 1.4 NA, OLYMPUS, Japan) was located just below the lower chamber. Bilayers were epi-illuminated by green and red

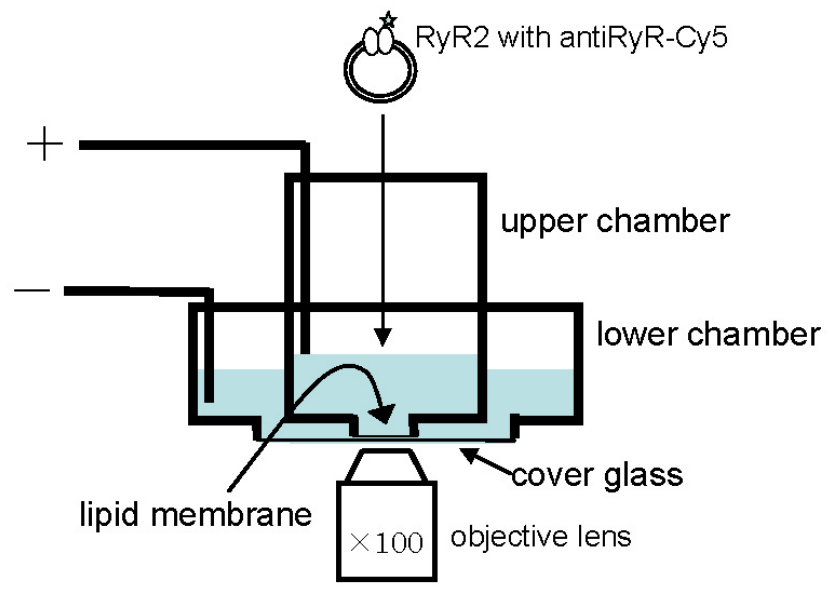

FIG. 2: Overview of the lipid bilayer formation apparatus. This apparatus consists of two chambers. Lipid bilayers are formed in a small hole (about $100 \mu$ m diameter) at the bottom of the upper chamber. The bottom surface of the bilayers touches the agarose-coated cover glass at the bottom of the lower chamber.

lasers (microgreen SLM laser, Uniphase, USA, He-Ne Red laser, Melles Griot, USA). Images were recorded using an ICCD camera, an image intensifier (VS4-1845, Video Scope, USA) and an electron bombarded CCD camera (C7190-20, Hamamatsu Photonics, Japan), and stored on digital videotape. Video sequences were imported and analyzed by a personal computer using image analysis software. Images were acquired every $33 \mathrm{~ms}$.

\section{E. Measurement conditions}

Lipids contained phosphatidylethanolamine (PE), phosphatidylserine (PS), and phosphatidylcholine (PC) at a 5:3:2 ratio $(50 \mathrm{mg} / \mathrm{ml}$ phospholipids in n-decane). $0.05 \% \beta$-BODIPY HPC (Molecular Probes, USA) was mixed with the lipid. The bilayer was formed by placing lipids at the hole of the upper chamber. Purified RyR2 with Cy5-conjugated antiRyR2 was added to the upper chamber. After forming the bilayer, $1 \mu \mathrm{M}$ annexin 5 was added to the lower chamber. Measurements were performed more than 30 minutes after the addition of annexin 5. The upper chamber solution contained 250 $\mathrm{mM} \mathrm{KCl,} 20 \mathrm{mM}$ Pipes $\mathrm{pH}$ 7, $0.1 \mathrm{mM} \mathrm{CaCl}_{2}$ and 0.1 mM EGTA and the lower chamber solution contained 250 $\mathrm{mM} \mathrm{KCl,} 20 \mathrm{mM}$ Pipes $\mathrm{pH} 7,1 \mathrm{mM} \mathrm{CaCl}_{2}$ and $0.1 \mathrm{mM}$ EGTA. $\beta$-BODIPY HPC and Cy 5 were exited with green $(534 \mathrm{~nm})$ and red $(633 \mathrm{~nm})$ lasers, respectively. Transbilayer currents were recorded and analyzed using a system comprised of an Axopatch 1B patch clamp amplifier, a Digidata 1200 interface, a PC computer and pClamp 9 software.

\section{F. Data analysis}

The mean square displacement $\left(<r^{2}>\right)$ averaged over a trajectory at each time interval $(\Delta t)$ was calculated from 


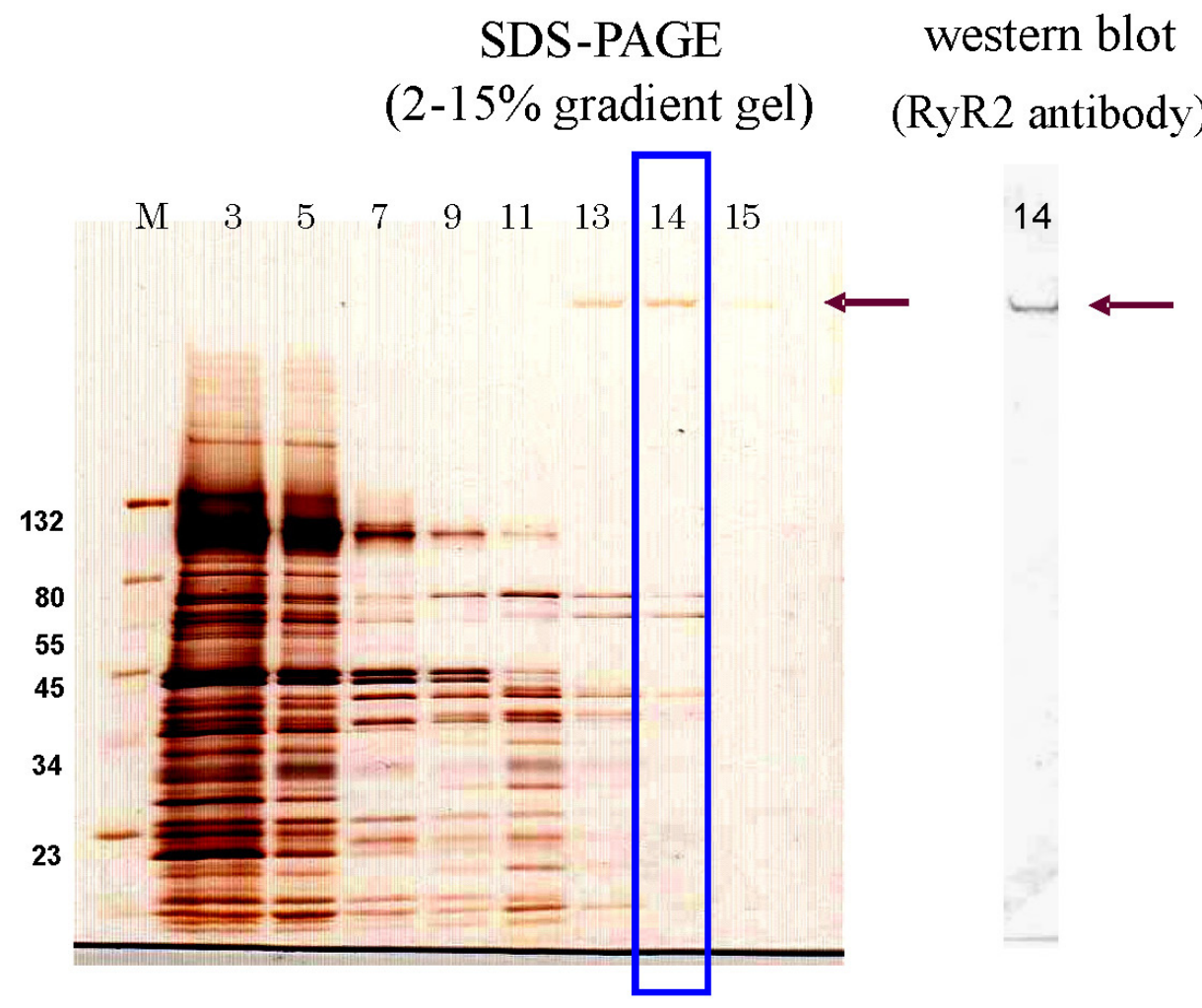

FIG. 3: SDS-PAGE and western blot analysis of purified RyR2. Fractionated sample was analyzed. M is a molecular marker $(\mathrm{kDa})$. Arrows point to the position of RyR2. We took No. 14 (blue rectangle) to perform western blot against RyR2.

TABLE I: The average values of the diffusion coefficients and reduction rates of lipid ( $\beta$-BODIPY HPC) and protein (RyR2 with antiRyR2-Cy 5).

\begin{tabular}{rcrr}
\hline \hline & - annexin $\left(10^{-8} \mathrm{~cm}^{2} / \mathrm{s}\right)$ & $1 \mu \mathrm{M}$ annexin $\left(10^{-10} \mathrm{~cm}^{2} / \mathrm{s}\right)$ & reduction rate \\
\hline B-BODIPY HPC & $11 \pm 6.7(\mathrm{n}=7)$ & $2.4 \pm 1.3(\mathrm{n}=8)$ & $1 / 450$ \\
RyR2 with antiRyR2-Cy 5 & $2.7 \pm 2.1(\mathrm{n}=9)$ & $2.6 \pm 2.0(\mathrm{n}=8)$ & $1 / 104$ \\
\hline \hline
\end{tabular}

the trajectory of a particle. The diffusion constant, D, was calculated from the slope of the $\Delta t-<r^{2}>$ plot by least-squares fitting.

\section{RESULTS}

\section{A. RyR2 purification}

Figure 3 shows SDS-PAGE and western blot of purified RyR2. The fractionated samples after the sucrose gradient were run on a $2-15 \%$ gradient gel and silver stained. Numbers of column fractions are shown in the upper side of Fig. 3A. Arrows indicate bands of RyR2. We took fraction No. 14 (blue rectangle) and then reconstituted the following western blot. Fig. 3B is the result of the western blot against RyR2 antibody. The presence of only one band in Fig. 3B indicates RyR2 antibody does not bind to the lower bands shown in the No. 14 fraction. This means that the fluorescence of antiRyR2-Cy5 indicates the presence of RyR2.
B. Annexin 5 decreases the diffusion of fluorescent lipids and RyR channels in the lipid bilayer

Figure 4 shows typical trajectories of single $\beta$-BODIPY HPC and RyR2 molecules in the absence and presence of 1 $\mu \mathrm{M}$ annexin 5 . Free lateral diffusion of $\beta$-BODIPY HPC in lipid bilayer is shown in Fig. 4A. The average value of the diffusion coefficients for $\beta$-BODIPY HPC in the absence of annexin 5 was $11 \pm 6.7 \times 10^{-8} \mathrm{~cm}^{2} / \mathrm{s}(\mathrm{n}=7)$. Figure $4 \mathrm{~B}$ is a typical trace of single $\beta$-BODIPY HPC in the presence of $1 \mu \mathrm{M}$ annexin 5 . The average value of the diffusion coefficient was $2.3 \pm 1.3 \times 10^{-10} \mathrm{~cm}^{2} / \mathrm{s}(\mathrm{n}=8)$. In the presence of $1 \mu \mathrm{M}$ annexin 5 , the diffusion coefficient decreased to $1 / 490$ th of its value in the absence of annexin 5. Figures $4 \mathrm{C}$ and $\mathrm{D}$ are typical traces of antiRyR2-Cy5 labeled RyR2 molecules. The average values of the diffusion coefficients of RyR2 labeled in the absence and in the presence of $1 \mu \mathrm{M}$ of annexin 5 were $2.7 \pm 2.1 \times 10^{-8} \mathrm{~cm}^{2} / \mathrm{s}$ $(\mathrm{n}=9)$ and $2.6 \pm 2.0 \times 10^{-10} \mathrm{~cm}^{2} / \mathrm{s}(\mathrm{n}=8)$, respectively. One micro molar of annexin 5 decreased the diffusion of labeled RyR2 to $1 / 104$ th of its value in the absence of annexin 5. The average values of the diffusion coefficients and reduction rates are shown in Table 1. 


\section{- annexin 5}

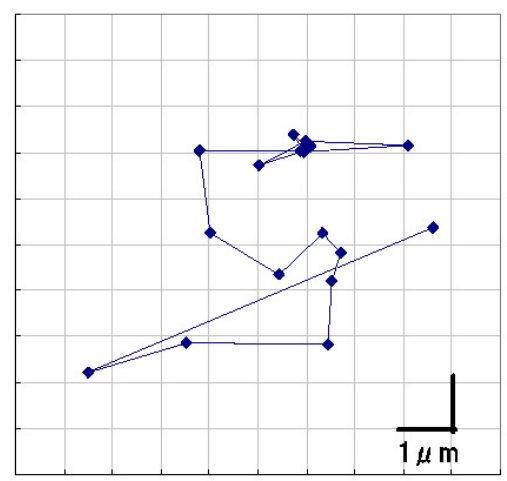

A

- annexin 5

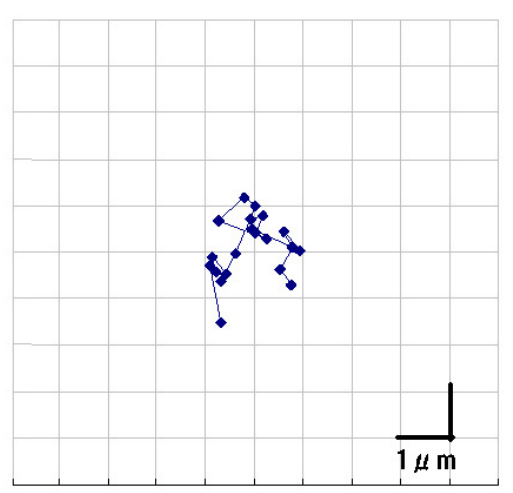

C

\section{$1 \mu \mathrm{M}$ annexin 5}

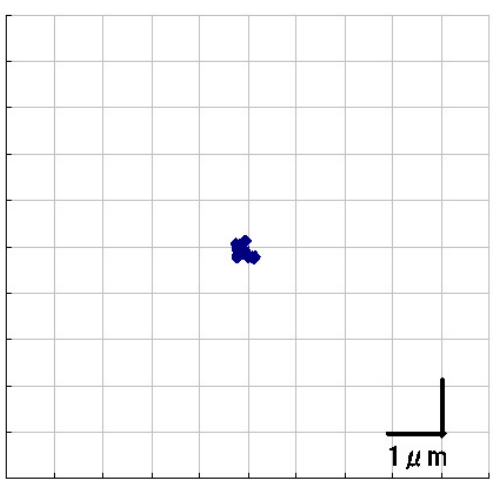

B

\section{$1 \mu \mathrm{M}$ annexin 5}

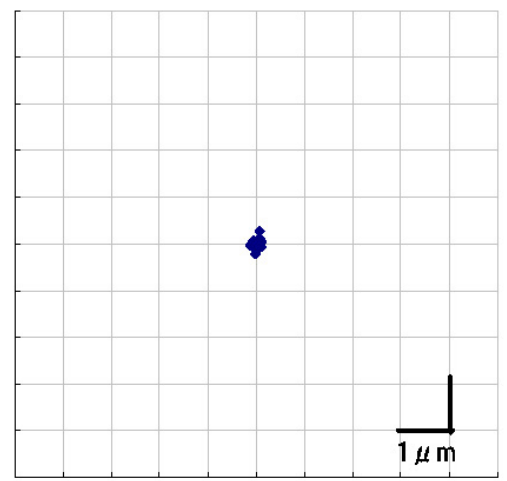

$\mathrm{D}$

FIG. 4: Trajectories of single $\beta$-BODIPY HPC and RyR2 molecules with antiRyR2-Cy5 in the lipid bilayer with and without 1 $\mu \mathrm{M}$ annexin 5. Top row is $\beta$-BODIPY HPC in the absence of annexin $5(\mathrm{~A})$ and in the presence of $1 \mu \mathrm{M}$ annexin 5 (B). Bottom row is labeled RyR2 in the absence of annexin $5(\mathrm{C})$ and in the presence of $1 \mu \mathrm{M}$ annexin $5(\mathrm{D})$. Data were acquired at $33 \mathrm{kHz}$. 20 points are shown. The time lag between two consecutive observations was $33 \mathrm{~ms}$. Lipids consisted of 5:3:2 PE/PS/PC (50 $\mathrm{mg} / \mathrm{ml}$ in decane). Annexin 5 was added to the lower chamber.

\section{Influence of annexin 5 on RyR2 properties}

The ability of annexin 5 to limit RyR2 diffusion could make it a useful tool for optical analyses of the function of RyR2 channels reconstituted in lipid bilayers. However, such use requires that RyR2 channel properties are not altered. Figure 5 shows the trace of RyR 2 in the absence and presence of $1 \mu \mathrm{M}$ annexin 5 , respectively. These figures indicate that annexin 5 does not so much change the electrophysiological properties of RyR2. Although extensive studies are needed to determine the influence of annexin 5, preliminary observation suggests that annexin 5 does not exert marked effects on RyR2 gating and activation.

\section{DISCUSSION}

By using an optical lipid bilayer system, we could observe single lipids and RyR2 molecules directly. The average value of the diffusion coefficients of $\mu$-BODIPY HPC in the absence of annexin 5 was $11 \pm 6.7 \times 10^{-8} \mathrm{~cm}^{2} / \mathrm{s}$ $(n=7)$. This value agreed well with the value for a single lipid moving freely in a membrane $[2,3]$. The average value of the diffusion coefficients of RyR2 labeled in the absence and in the presence of $1 \mu \mathrm{M}$ of annexin 5 were $2.7 \pm 2.1 \times 10^{-8} \mathrm{~cm}^{2} / \mathrm{s}(\mathrm{n}=9)$ and $2.6 \pm 2.0 \times 10^{-10} \mathrm{~cm}^{2} / \mathrm{s}$ $(n=8)$, respectively. These values are in the same order as the value of the bilayer experiment performed by using single RyR2 [5]. Bright spots in the presence of annexin 5 appeared nearly immobile in the microscopic field. Therefore annexin 5 may be an effective way to immobilize a channel protein in a lipid bilayer.

The most remarkable feature of annexins, including annexin 5 , is the ability to associate with biological membranes and lipid bilayers through $\mathrm{Ca}^{2+}$ dependent binding to anionic phospholipids, such as PS [7]. Recently structural studies have led to various models to explain how annexins bind to a lipid bilayer [14-16]. These include annexin 5 burying itself in the monolayer of the membrane; forming transmembrane structure accessible to both sides of the bilayer; binding to the head of the lipid. In our study, stopped spots and rapidly moving spots of $\beta$-BODIPY HPC are simultaneously observed in 


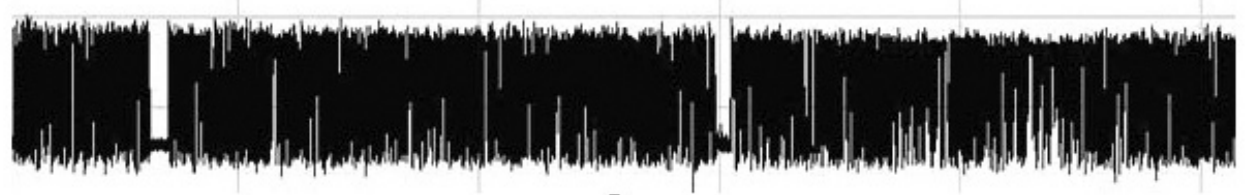

A

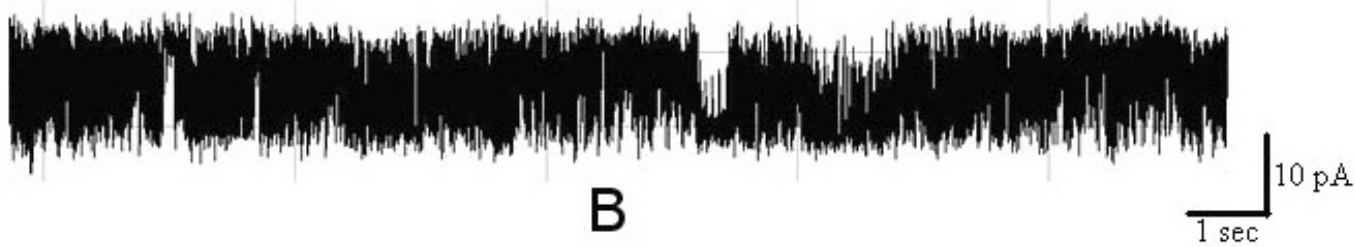

FIG. 5: Typical traces of single RyR2 molecules recording with (A) and without (B) annexin 5 .

the presence of $1 \mu \mathrm{M}$ annexin 5 in the lower chamber (data not shown). Thus in our experiments, annexin 5 decreases the diffusion of both lipids on only one side of lipid bilayer. From this observation, we conclude that annexin 5 does not penetrate the lipid bilayer.

We found that annexin 5 decreases the diffusion of lipid bilayers and caused minimal change to RyR2 properties. This method enables us to immobilize channel proteins in lipid bilayers and monitor the single channel proteins for a long time. This is a preliminary step to simultaneously observe the optical and electrical properties of ryanodine binding to RyR2. The aim of this experiment is to acquire information about the relationship between ligand binding to the receptor and the change of function. In order to acquire further information, FRET or other imaging techniques are required. These simultaneous examinations will reveal dynamic conformations of the protein.
[1] S. Weiss, Science 283, 1676 (1999).

[2] T. Ide, and T. Yanagida, Biochem. Biophys. Res. Commun. 265, 595 (1999).

[3] T. Ide, Y. Takeuchi, T. Aoki, and T. Yanagida, Jpn J. Physiol. 52, 429 (2002).

[4] S. Greg, G. O. Harms, M. Montal, B. D. Thrall, S. D. Colson, and H. P. Lu, Biophys. J. 85, 1826 (2003).

[5] S. Peng, N. G. Publicover, G. J. Kargacin, D. Duan, J. A. Airey and J. L. Sutko, Biophys. J. 86, 134 (2004).

[6] S. Peng, N. G. Publicover, J. A. Airey, J. E. Hall, H. T. Haigler, D. Jiang, S. R. W. Chen and J. L. Sutko, Biophys. J. 86, 145 (2004).

[7] V. Gerke, and S. E. Moss, Physiol. Rev. 82, 331 (2002).

[8] M. A. Swairjo, and B. A. Seaton, Annu. Rev. Biophys. Biomol. Struct. 23, 193 (1994).

[9] C. E. Creutz, Science 258, 924 (1992).
[10] J. Sopkova, M. Renouard and A. Lewit-Bentley, J. Mol. Biol. 234, 816 (1993).

[11] K. Anderson, F. A. Lai, Q. Y. Liu, E. Rousseau and G. Meissner, J. Biol. Chem. 264, 1329 (1989).

[12] H. B. Lee, L. Xu and G. Meissner, J. Biol. Chem. 269, 13305 (1994).

[13] U. Rescher and V. Gerke, J. Cell. Sci. 117, 2631 (2004).

[14] A. S. Ladokhin and H. T. Haigler, Biochemistry 44, 3402 (2005).

[15] F. Oling, W. Bergsma-Schutter, and A. Brisson, J. Struct. Biol. 133, 55 (2001).

[16] Y. Mo, B. Campos T. R. Mealy, L. Commodore J. F. Head, J. R. Dedman and B. A. Seaton, J. Biol. Chem. 278, 2437 (2003). 